\title{
Finite-Size Effects on Nucleation in a First-Order Phase Transition
}

\author{
Eduardo S. Fraga ${ }^{1}$ and Raju Venugopalan ${ }^{2}$ \\ ${ }^{1}$ Instituto de Física, Universidade Federal do Rio de Janeiro \\ C.P. 68528, Rio de Janeiro, RJ 21941-972, Brazil \\ ${ }^{2}$ Department of Physics and RIKEN-BNL Research Center, \\ Brookhaven National Laboratory, Upton, NY 11973-5000, USA
}

(October 30, 2018)

We discuss finite-size effects on homogeneous nucleation in first-order phase transitions. We study their implications for cosmological phase transitions and to the hadronization of a quark-gluon plasma generated in high-energy heavy ion collisions. Very general arguments allow us to show that the finite size of the early universe has virtually no relevance in the process of nucleation and in the growth of cosmological bubbles during the primordial quark-hadron and the electroweak phase transitions. In the case of high-energy heavy ion collisions, finite-size effects play an inportant role in the late-stage growth of hadronic bubbles.

\section{INTRODUCTION}

First-order phase transitions and the kinetic phenomena associated with the process of phase conversion through the nucleation of bubbles or spinodal decomposition are present in almost all realms of physics [1]. Usually, the interesting physical quantities are the time scales related to the transient regime, since they determine its relative importance. However, one cannot often disentangle those scales from other scales of the problem, such as the different length scales and, in particular, the finite size of the system under consideration.

Despite the success of finite-size scaling in the study of equilibrium critical phenomena [2], a systematic study of finite size effects has rarely been conducted in the case of metastable decays and other nonequilibrium processes (see Ref. [3] and references therein). In particular, a careful analysis of possible modifications of the usual picture of nucleation in a thermally-driven first-order phase transition due to the finite size of the system seems to be lacking.

In this paper, we discuss finite size effects on the dynamics of homogeneous nucleation in a first-order temperature-driven transition. In particular, we consider the case of cosmological phase transitions in the early universe [4], and that of a quark-gluon plasma (QGP) decay into hadronic matter in a high-energy heavy ion collision $[5,6]$. The former might provide sensible mechanisms to explain the baryon number asymmetry in the universe and primordial nucleosynthesis $[7,8]$, whereas the latter is expected to be observed [9] at BNL Relativistic Heavy Ion Collider (RHIC). The length and time scales involved in each of these cases differ by several orders of magni- tude. We will argue that, despite statements to the contrary in the literature, the finite size of the early universe plays no important role in the process of homogeneous nucleation during the quark-hadron and the electroweak cosmological phase transitions. For an expanding QGP plasma, finite size effects might prove to be relevant if the plasma is formed in relativistic heavy ion collisions.

In the usual description of homogeneous nucleation [1], there are two ways in which the finite size of the system can affect the formation and evolution of bubbles and, consequently, the dynamics of phase conversion. Firstly, one has to consider the effects on the nucleation rate and the early stage growth of the bubbles. As will be shown below, this correction comes about through an intrinsic uncertainty in the determination of the supercooling undergone by the system. For the cases considered here, it brings only minor modifications to a description which assumes an infinite system. The second and, in general, most important finite-size effect is its influence on the domain coarsening process, or late-stage growth of the bubbles. The relevant length scales here are given by the typical size of the system, the radius of the critical bubble and the correlation length. One has to compare these scales, in each case of interest, to measure the relevance of possible corrections due to the finite size of the system.

The arguments presented in this paper are based solely on analytic considerations and simple estimates. To obtain precise results, one should perform numerical simulations. Lattice methods have been successfully applied to the study of homogeneous nucleation in different contexts $[3,10,11]$. A systematic lattice investigation of bubble nucleation and spinodal decomposition, including finite-size effects, in the case of high-energy heavy ion collisions is certainly important and will be reported in a future publication [12].

The paper is organized as follows. In Section II, we present a short review of relevant aspects of homogeneous nucleation that are important for our analysis. In section III, we discuss the effects of the finite size of the system in general, and apply the results to the cases mentioned above. Section IV contains our conclusions and outlook. 


\section{HOMOGENEOUS NUCLEATION IN A FIRST-ORDER PHASE TRANSITION}

In a continuum description of a first-order phase transition, it is convenient to make use of a coarse-grained free energy, $F$, which is expected to have the familiar LandauGinzburg form with temperature-dependent coefficients [1]. (In the case of QCD, such a free energy can be obtained, for instance, from the one-loop effective potential of a linear sigma model coupled to quarks [13,14].) For temperatures between the critical temperature, $T_{c}$, and a temperature that characterizes the spinodal region, $T_{s p}$, the minimum corresponding to the symmetric phase is metastable, and gradually disappears as the system approaches $T_{s p}$, where the symmetric phase becomes unstable. In other words, at $T=T_{s p}$, the barrier that separates the metastable symmetric phase minimum from the true vacuum, within this temperature range, vanishes. The driving mechanism for the phase transition above the spinodal temperature is the nucleation of large localized domains, or bubbles, of the true vacuum phase, inside the so-called false vacuum or metastable phase, via thermal activation. Below $T_{s p}$, one has the phenomenon of spinodal decomposition, where the transition is driven by long-wavelength small fluctuations [1]. An expanding system will probe these two regions at a pace given by its rate of expansion. The investigation of the time scale for thermal nucleation relative to that for the expansion, to evaluate the role played by nucleation in the phase conversion, is therefore an interesting issue (see, for instance, Ref. [14]). However, for a more complete and consistent analysis, one also has to incorporate the effects due to the finite size of the system under investigation.

The nucleation rate, namely, the probability per unit volume, per unit time, to form a critical bubble, can be expressed as $\Gamma=\mathcal{P} e^{-F_{b} / T}$, where $F_{b}$ is the free energy of a critical bubble. The prefactor $\mathcal{P}$, discussed further below, measures statistical and dynamical fluctuations about the saddle point of the Euclidean action in functional space. The critical bubble, with radius $R=R_{c}$, is a field configuration given by a radially symmetric, static solution of the Euler-Lagrange field equations corresponding to an exact balance between competing volume and surface energy contributions. The critical bubble is unstable with respect to small changes of its radius; for subcritical bubbles, $R<R_{c}$, the surface energy dominates, and the bubble shrinks and vanishes, while for supercritical bubbles, $R>R_{c}$, the volume energy dominates, and the bubble grows driving the decay process.

It is convenient to write the prefactor $\mathcal{P}$ as a product of the bubble's growth rate and a factor proportional to the ratio of the determinant of the fluctuation operator around the bubble configuration relative to that around the homogeneous metastable state [15]. For the relativistic case, in the thin-wall limit, we have [16]:

$$
\mathcal{P}=\frac{16}{3 \pi}\left(\frac{\sigma}{3 T}\right)^{3 / 2} \frac{\sigma \eta R_{c}}{\xi^{4}(\Delta \omega)^{2}} .
$$

Here, $\eta$ and $\xi$ are respectively the shear viscosity and the correlation length in the symmetric phase and $\Delta \omega$ is the enthalpy density difference between the two phases. $\sigma$ is the surface tension of the interface, which is related to the critical radius and the difference in pressure between the mestastable phase and the true vacuum, $\Delta p$, as $R_{c}=$ $2 \sigma / \Delta p$. The thin-wall limit is a good approximation in the limit of small supercooling, $\theta=1-T / T_{c} \ll 1$. In this limit, the decay rate can be written as

$$
\Gamma=\mathcal{P} \exp \left(-\frac{4 \pi \sigma}{3 T} R_{c}^{2}\right)=\mathcal{P} \exp \left(-\frac{16 \pi}{3} \frac{\sigma^{3}}{T_{c} \ell^{2} \theta^{2}}\right)
$$

where $\ell$ is the latent heat density. For increasing values of the supercooling, $\theta$, the argument of the exponential decreases and the decay rate increases. Equivalently, the critical radius becomes smaller, so that it is easier to nucleate supercritical bubbles. The only way in which the finite size of the system can affect the decay rate is through its influence on the supercooling. (Of course, one has to check whether the size of the system allows for the presence of at least one critical bubble, including the wall that separates the two phases, or not.)

\section{FINITE-SIZE EFFECTS}

\section{A. Coarse-grained free energy}

Before considering the effects of the finite size of the system on the nucleation process, let us move one step backwards to examine a point that will be relevant for the following, namely, the definition of metastable states in equilibrium statistical mechanics. Since the complete partition function is dominated by configurations that minimize the free energy and correspond to equilibrium states, it does not allow for the existence of metastable branches in the thermodynamic limit. One solution to this difficulty consists in constraining the phase space and computing restricted partition functions. In order to define an optimal cut, one has to resort to physical arguments [1].

Such a procedure can be implemented as follows [17]. The system of size $L$ is divided into cells of linear size $\lambda_{c g}$ centered at positions $\vec{x}$. The length scale which defines the coarse-graining of the system, $\lambda_{c g}$, should be appreciably larger than the underlying lattice spacing $a$. Moreover, within each cell, the relevant order parameter, $\phi$, should vary smoothly in space and the equilibration time of the system should be much faster than the processes under consideration. This allows us to adopt a 
continuum order parameter, $\phi(\vec{x}, t)$, defined as an average over each cell.

However, by construction, the coarse-grained free energy will depend on the scale $\lambda_{c g}$ and, as mentioned previously, one has to appropriately constrain the phase space to allow for metastable states. If $\lambda_{c g}$ is small enough as compared to the correlation length, $\xi$, phase separation within a cell cannot occur and one can define a coarse-grained free energy, $F$, such as the one we considered in the discussion of Section II. The condition $\lambda_{c g}<<\xi$ therefore represents the physically motivated restriction to the partition function that allows for a well-defined coarse-grained description of metastable states. As a corollary, increasing the value of the coarsegraining length relative to the correlation length would incorporate more and more fluctuation modes, which were excluded by this ultraviolet cut-off. The free energy $F$ would then flatten out completely in the limit $\lambda_{c g} / \xi \rightarrow \infty$, thereby approaching the equilibrium description with no metastable branch [18]. For a system to be characterized by a well-defined Landau-Ginzburg coarse-grained free energy one therefore requires a clean separation of scales: $a<<\lambda_{c g}<<\xi<<L$.

\section{B. Nucleation rate}

The only effect that might be relevant to the nucleation rate, $\Gamma$, is the rounding of singularities, since they will affect the degree of supercooling. The relevant physical quantity can be calculated in a finite-size scaling framework [19], resulting in the following expression:

$$
\frac{\Delta T_{\text {round }}}{T_{c}} \approx \frac{2 T_{c}}{\ell L^{d}}
$$

where $L$ is the typical length scale of the system and $d$ is the number of dimensions. The quantity $\Delta T_{\text {round }}$ is a measure of the smoothening of singularities due to the finite size of the system [20]. It corresponds to the (now non-vanishing) width in temperature of the region where the energy density of the system suffers an abrupt jump, around $T=T_{c}$ [21]. This phenomenon enters the decay rate as a minimum value for $\theta$ which reflects the uncertainity in the supercooling due to the finite size of the system. This conclusion should be contrasted with the arguments presented in Ref. [22], particularly in the case of cosmological phase transitions [23].

For phase transitions in the early universe, $L$ is given by the radius of the universe at a given time (or temperature). Going back to very early times, $t \sim 10^{-4} \mathrm{~s}$ and $T \sim 10^{12} \mathrm{~K} \sim 10^{-1} \mathrm{GeV}$, we can use the elementary particle model [24] to obtain an approximate equation of state (ideal gas)

$$
3 p \approx \epsilon \approx \frac{\pi^{2}}{30} N(T) T^{4}
$$

where $N(T)=N_{\text {bosons }}(T)+(7 / 8) N_{\text {fermions }}(T)$ is the total number of degrees of freedom, $\epsilon$ is the energy density and $p$ is the pressure. From Einstein's field equations, one can derive the relation between time and energy density in the early universe, which links the age of the universe and its temperature in the following way

$$
t \approx\left(\frac{3}{32 \pi G \epsilon}\right)^{1 / 2} \approx \frac{1}{4 \pi}\left(\frac{45}{\pi N(T)}\right)^{1 / 2} \frac{M_{P l}}{T^{2}}
$$

where $M_{P l}$ is the Planck mass and $G$ is Newton's gravitational constant. The radius of the universe, as given by the particle horizon in a Robertson-Walker spacetime [25], $d_{h}(t)=t /(1-n)$, where the scale factor grows as $a(t) \sim t^{2 / 3(1+w)}=t^{n}$ and $p=w \epsilon$, has the following form:

$$
L_{u n i v}(T) \approx \frac{1}{4 \pi}\left(\frac{1}{1-n}\right)\left(\frac{45}{\pi N(T)}\right)^{1 / 2} \frac{M_{P l}}{T^{2}} .
$$

For the assumed equation of state, $w=1 / 3$ and $n=$ $1 / 2$. If we enter temperature in $\mathrm{GeV}$, the typical length scale of the early universe is given, in fermi units, by $L_{\text {univ }}(T) \approx \mathcal{A} / T^{2} \sqrt{N(T)}$, where $\mathcal{A}=1.45 \times 10^{18}$.

It is clear that, due to the large factor $\sim 10^{18}$ (coming from $\left.M_{P l}\right), L_{u n i v}$ will be of importance for supercooling only for extremely high values of the temperature. Since $N(T) \approx 50$ for the cases of the QCD and the electroweak phase transitions, one would need $T \sim 10^{8} \mathrm{GeV}$ to be subject to appreciable finite-size effects on the nucleation rate. Therefore, since $T_{c}^{Q C D} \sim \mathcal{O}\left(10^{-1}\right) \mathrm{GeV}$ and $T_{c}^{E W} \sim \mathcal{O}\left(10^{2}\right) \mathrm{GeV}$, these effects are negligible in such cases. They might prove to be relevant in the case of GUT's transitions, where the critical temperature will be much higher.

For the first order transition of a quark-gluon plasma into a hadronic gas in a high-energy heavy ion collision, one can easily estimate the role of finite-size effects on the nucleation rate. The parameters entering Eq. (3) are approximately given by $T_{c} \approx 150 \mathrm{MeV}$ [26], $\ell \approx 4 B \approx 4 \times(150 \mathrm{MeV})^{4}$, where $B$ is the bag constant, and $L \approx 10 \mathrm{fm}$. The minimal amount of supercooling undergone by the plasma is then negligible, of the order of $10^{-3}$. (For more conservative, smaller values of $L$, the minimum value of $\theta$ is still very small.) The system can in principle probe the entire domain in $\theta$, and there are no constraints on homogeneous nucleation from this side. The question of how fast the system probes the nucleation region, before reaching the spinodal regime, as compared to the nucleation rate was discussed in Ref. $[14,27,28]$. Recently, it has been speculated that the RHIC data suggest an explosive hadron production due to a rapid variation of the effective potential for QCD close to $T_{c}$. The theoretical reasoning is based on the results of the Polyakov Loop Model [29] for the deconfining phase transition, which lead to a very fast spinodal decomposition regime [30]. 


\section{Late-stage growth}

After the nucleation of a given supercritical bubble, it will grow with a certain velocity. The set of all supercritical bubbles created integrated over time will eventually drive the complete phase conversion in a finite system. The scales that determine the importance of finite-size effects are the typical linear size of the system, the radius of the critical bubble and the correlation length. For the reasons discussed in the last subsection, the case of cosmological phase transitions is to an excellent approximation free from finite-size corrections. The enormous numerical value of the Planck mass washes out every other scale. Therefore, throughout this subsection we will address the quark-gluon-hadron phase transition in heavy ion collisions.

For definiteness, let us assume our system is characterized by a coarse-grained free energy of the form

$$
F(\phi, T)=\int d^{d} x\left[\frac{1}{2}(\nabla \phi)^{2}+U(\phi, T)\right],
$$

where $U(\phi, T)$ is some Landau-Ginzburg potential whose coefficients depend on the temperature, and $\phi(\vec{x}, t)$ is a scalar field [31]. For the cases to be considered in this paper, the order parameter, $\phi$, is not a conserved quantity, and its evolution is given by the time-dependent LandauGinzburg equation [1]

$$
\frac{\partial \phi}{\partial t}=-\gamma \frac{\delta F}{\delta \phi}=\gamma\left[\nabla^{2} \phi-U^{\prime}(\phi, T)\right],
$$

where $\gamma$ is the response coefficient which defines a time scale for the system. Equation (8) is a standard reactiondiffusion equation, and describes the approach to equilibrium [32].

If $U(\phi, T)$ is such that it allows for the existence of bubble solutions (taken to be spherical for simplicity), then supercritical (subcritical) bubbles expand (shrink), in the thin-wall limit, with the following velocity:

$$
\frac{d R}{d t}=\gamma(d-1)\left[\frac{1}{R_{c}}-\frac{1}{R(t)}\right],
$$

where $R_{c}=(d-1) \sigma / \Delta F$ and $\Delta F$ is the difference in free energy between the two phases. Equation (9) is an example of the Allen-Cahn equation [1], which relates the velocity of a domain wall to the local curvature. The response coefficient, $\gamma$, can be related to some characteristic collision time as will be done later.

The description of the late-stage domain coarsening is given by the Kolmogorov-Avrami theory [1], which contains the following assumptions: (i) bubbles grow without substantial deformation and are uncorrelated; (ii) the nucleation rate is a constant; (iii) the bubble growth velocity is constant, $v=d R / d t=\gamma(d-1) / R_{c}$ (limit $R \rightarrow \infty)$. The quantity which is usually computed is the volume fraction of the stable phase.
However, one can measure the importance of finite-size effects for the case of heavy-ion collisions by comparing, for instance, the asymptotic growth velocity $\left(R>>R_{c}\right)$ for nucleated hadronic bubbles to the expansion velocity of the plasma. In the Bjorken picture, one assumes that the central rapidity region exhibits longitudinal expansion, so that $z(t)=v_{z} t$, where $v_{z}$ is the collective fluid velocity. Conservation of total entropy leads to adiabatic expansion and the following cooling behavior: $\left(t / t_{i}\right)=\left(T_{i} / T\right)^{3}$. The typical length scale of the expanding system is then

$$
L(T) \approx\left(v_{z} t_{c}\right)\left(\frac{T_{c}}{T}\right)^{3}=L_{0}\left(\frac{T_{c}}{T}\right)^{3},
$$

where $L_{0} \equiv L\left(T_{c}\right)$ is the initial linear scale of the system for the nucleation process which starts at $T \leq T_{c}$.

The relation between time and temperature provided by the cooling law that emerges from the Bjorken picture suggests the comparison between the following "velocities":

$$
v_{b} \equiv \frac{d R}{d T}=-\left(\frac{3 b \ell L_{0}}{2 v_{z} \sigma T_{c}^{2}}\right)\left(\frac{T_{c}}{T}\right)^{5}\left(1-\frac{T}{T_{c}}\right),
$$

the asymptotic bubble growth "velocity", and the plasma expansion "velocity"

$$
v_{L} \equiv \frac{d L}{d T}=-\frac{3 L_{0}}{T_{c}}\left(\frac{T_{c}}{T}\right)^{4} .
$$

The quantity $b$ is a number of order one to first approximation, and comes about in the estimate of the phenomenological response coefficient $\gamma(T) \approx b / 2 T$ (see Ref. [33] for details).

Using the numerical values adopted in the previous section and $\sigma / T_{c}^{3} \sim 0.1$, we obtain

$$
\frac{v_{b}}{v_{L}} \approx \frac{20}{v_{z}}\left(\frac{T_{c}}{T}-1\right)
$$

One thus observes that the bubble growth velocity becomes larger than the expansion velocity for a supercooling of order $\theta \approx v_{z} / 20 \leq 5 \%$. A simple estimate points to a critical radius larger than $1 \mathrm{fm}$ at such values of supercooling (see also [14]). Therefore, finite-size effects appear to be an important ingredient in the phase conversion process right from the start in the case of high-energy heavy-ion collisions.

\section{CONCLUSIONS AND OUTLOOK}

We have studied the effects of the finite size of the system on the nucleation of bubbles in thermally-driven first-order phase transition. The physical situations we considered were those of the cosmological transitions in 
the early universe and the hadronization of a quark-gluon plasma after a high-energy heavy ion collision. We have shown, on very general grounds, that during the quarkhadron and the electroweak cosmological transitions the universe is large enough so that one is safe to approximate it by an infinite system. Corrections due to its finite size are negligible. For the case of heavy ions, although the influence on the nucleation rate is also small, effects related to domain coarsening and late-stage growth are important and should be taken into account in a detailed study.

In order to address the question of hadronization after a first-order transition, one should then perform finitesize real-time lattice simulations. One would thereby avoid the drawbacks implied by analytical approximations, such as the thin-wall hypothesis. Moreover, one would be able to control the behavior of domains and study, for instance, scaling properties [12]. It is also interesting to study the hydrodynamics of nuclear matter at chiral limit as a phenomenological description of the chiral transition in an expanding quark-gluon plasma [34-37]. Results in this direction will be presented elsewhere.

We thank A. Dumitru, R. Gavai, S. Gupta and A. Krasnitz for fruitful discussions. E.S.F. thanks the members of LPT (Orsay), where part of this work has been done, for their kind hospitality. E.S.F. is partially supported by FAPERJ and FUJB/UFRJ. R.V. is supported by the U.S. Department of Energy under Contract No. DE-AC02-98CH10886 and by the RIKEN-BNL Research Center at BNL.

[1] J. D. Gunton, M. San Miguel and P. S. Sahni in Phase Transitions and Critical Phenomena (Edited by C. Domb and J. L. Lebobowitz, Academic Press, London, 1983), vol. 8; K. Binder, Rep. Prog. Phys. 50, 783 (1987). J. S. Langer in Solids far from equilibrium (Edited by C. Godrèche, Cambridge University Press, 1992); A. J. Bray, Adv. Phys. 43, 357 (1994).

[2] N. Goldenfeld, Lectures on Phase Transitions and the Renormalization Group - Frontiers in Physics vol. 85 (Perseus Press, 1992).

[3] P. A. Rikvold and B. M. Gorman in Annual Reviews of Computational Physics I (Edited by D. Stauffer, World Scientific, Singapore, 1994).

[4] For a recent review, see D. Boyanovsky, hep-ph/0102120.

[5] E.V. Shuryak, Phys. Rept. 61, 71 (1980); K. Kajantie and L. McLerran, Ann. Rev. Nucl. Part. Sci. 37, 293 (1987); B. Müller, Rept. Prog. Phys. 58, 611 (1995); J. Harris and B. Müller, Ann. Rev. Nucl. Part. Sci. 46, 71 (1996); S.A. Bass, M. Gyulassy, H. Stöcker and W. Greiner, J. Phys. G25, R1 (1999).

[6] L. P. Csernai and J. I. Kapusta, Phys. Rev. Lett. 69, 737
(1992); J. I. Kapusta, A. P. Vischer and R. Venugopalan, Phys. Rev. C 51, 901 (1995); J. I. Kapusta and A. P. Vischer, ibid. 52, 2725 (1995); E. E. Zabrodin, L. Bravina, H. Stocker and W. Greiner, ibid. 59, 894 (1999); P. Shukla, A. K. Mohanty, S. K. Gupta and M. Gleiser, ibid. 62, 054904 (2000).

[7] M. Trodden, Rev. Mod. Phys. 71, 1463 (1999).

[8] R. A. Malaney and G. J. Mathews, Phys. Rept. 229, 145 (1993).

[9] Proceedings of Quark Matter 2001, to appear in Nucl. Phys. A; http://rhic.bnl.gov/qm2001/.

[10] S. Gupta, Phys. Lett. B 325, 418 (1994).

[11] G. D. Moore and K. Rummukainen, Phys. Rev. D 63, 045002 (2001). G. D. Moore, K. Rummukainen and A. Tranberg, JHEP 0104, 017 (2001).

[12] E. S. Fraga, A. Krasnitz and R. Venugopalan, work in progress.

[13] O. Scavenius and A. Dumitru, Phys. Rev. Lett. 83, 4697 (1999).

[14] O. Scavenius, A. Dumitru, E. S. Fraga, J. T. Lenaghan and A. D. Jackson, Phys. Rev. D 63, 116003 (2001).

[15] J. S. Langer, Ann. Phys. 41, 108 (1967); ibid. 54, 258 (1969); ibid. 65, 53 (1971); J. S. Langer and L. A. Turski, Phys. Rev. A 8, 3230 (1973); L. A. Turski and J. S. Langer, ibid. 22, 2189 (1980).

[16] L. P. Csernai and J. I. Kapusta, Phys. Rev. D 46, 1379 (1992); R. Venugopalan and A. P. Vischer, Phys. Rev. E 49, 5849 (1994).

[17] J. S. Langer, Physica 73, 61 (1974).

[18] Note that this unphysical limit should not be confused with the thermodynamic limit of $L \rightarrow \infty$.

[19] K. Binder and D. P. Landau, Phys. Rev. B 30, 1477 (1984). M. S. S. Challa, D. P. Landau and K. Binder, ibid. 34, 1841 (1986).

[20] See C. Spieles, H. Stöcker and C. Greiner, Phys. Rev. C 57, 908 (1998) for a discussion of how the equation of state is changed in the case of the quark-hadron transition in a finite QGP.

[21] There is also a shift in the value of the critical temperature as a result of the finite size of the system [19]. Here, $T_{c}$ denotes the corrected value.

[22] B. Banerjee and R. V. Gavai, Phys. Lett. B 293, 157 (1992); Mod. Phys. Lett. A 11, 615 (1996).

[23] The authors of Ref. [22] interpret $\Delta T_{\text {round }} / T_{c}$ as the maximum amount of supercooling that can be reached in a finite system. A consequence is that Eq. 3 then severely constrains homogeneous nucleation in the case of the cosmological phase transitions. We argue that the situation in the case of cosmological transitions is precisely the opposite: any amount of supercooling above a (very small) minimum value is allowed and homogeneous nucleation in cosmological phase transitions is viable.

[24] S. Weinberg, Gravitation and Cosmology (John Wiley \& Sons, 1972).

[25] E. W. Kolb, M. S. Turner, The Early Universe (Perseus, 1993).

[26] F. Karsch, hep-ph/0103314.

[27] E. E. Zabrodin, L. V. Bravina, L. P. Csernai, H. Stocker and W. Greiner, Phys. Lett. B 423, 373 (1998).

[28] P. Shukla and A. K. Mohanty, Phys. Rev. C 64, 054910 (2001). 
[29] R. D. Pisarski, Phys. Rev. D 62, 111501 (2000).

[30] O. Scavenius, A. Dumitru and A. D. Jackson, Phys. Rev. Lett. 87, 182302 (2001).

[31] It is implicit in our discussion that we restrict ourselves to the case in which the order parameter is a scalar $(N=1$ in the language of critical phenomena), and that we assume that there is a maximum of two coexisting thermodynamic phases.

[32] In order to include the effect of fluctuations, one should add a Langevin noise term in (8).

[33] K. Kajantie, Phys. Lett. B 285, 331 (1992).

[34] D. T. Son, Phys. Rev. Lett. 84, 3771 (2000).

[35] I. N. Mishustin and O. Scavenius, Phys. Rev. Lett. 83, 3134 (1999).

[36] K. Paech, H. Stöcker and A. Dumitru, nucl-th/0302013.

[37] C. Aguiar, E. S. Fraga and T. Kodama, work in progress. 